\title{
MODELO ITERATIVO PARA ESTIMATIVA DA EVAPOTRANSPIRAÇÃO DE REFERÊNCIA PROVÁVEL
}

\author{
S. MARQUES Jr'; J.C.C. SAAD ${ }^{2}$; M.V.T. DE MOURA ${ }^{3}$ \\ 'Depto. Engenharia Rural, - CCANUSC, CP. 476, CEP 88040-900- Florianópolis - SC. \\ 2 Depto. Engenharia Rural - .FCA-UNESP, CEP: 18618-000-Botucatu, SP. \\ ${ }^{3}$ Secretaria da Agricultura e Abastecimento do Rıo Grande do Norte.
}

RESUMO: O presente trabalho teve por objetivo a apresentação de um modelo iterativo para utilização em microcomputadores capaz de estimar valores de evapotranspiração de referência em diversos níveis de probabilidade, a partir de uma série de dados. $O$ procedimento matemático envolvido na técnica iterativa empregada relaciona-se à utilização das funçōes de densidade gama incompleta e beta. Para tanto, foram utilizados dados quinzenais de evapotranspiração do mês de março, a partir de uma série de 30 anos para a região de Piracicaba-SP. Através do teste de Kolmogorov-Smirnov, verificou-se que dados estimados através do modelo apresentaram alto grau de ajuste com dados relatados em literatura, justificando assim sua utilização.

Descritores: evapotranspiraçāo, modelo iterativo, função beta

\section{ITERATIVE MODEL TO ESTIMATE PROBABLE REFERENCE EVAPOTRANSPIRATION}

\begin{abstract}
This paper presents an iteractive model for microcomputers to study the distribution of reference evapotranspiration, using beta and incomplete gamma density functions. For this purpose, two weekly data of reference evapotranspiration were used, collected in March during 30 years, for Piracicaba-SP, Brazil. Using the KolmogorovSmimov model, it was observed that estimated data showed to be closely related to experimental data found in literature. justifying its use.
\end{abstract}

Key Words: evapotranspiration, iteractive model, beta distribution

\section{INTRODUÇÃO}

No planejamento e desenvolvimento de sistemas que envolvam o manejo da água, o parâmetro "evapotranspiração" destaca-se como um dos componentes de maior importância. Este fato gerou, ao longo do tempo, uma contínua necessidade para obtenção de dados desse fator meteorológico, resultando no surgimento de inúmeros métodos de estimativa (JENSEN, 1974).

Entretanto, a grande variabilidade de valores assumidos pelos parâmetros meteorológicos que condicionam o ciclo hidrológico, acarretam, como comentam SAAD \& SCALOPPI (1988), considerável dispersão dos valores calculados de evapotranspiração, sugerindo uma análise de distribuição de frequências dos valores estimados .

Neste caso específico, os níveis de probabilidade a serem utilizados devem ser baseados em uma análise econômica onde seja considerado, por exemplo, a redução de produção e custos de uma cultura qualquer, quando da consideração de um valor estimado de evapotranspiração determinado à menores níveis de significância.
O objetivo do presente trabalho é a apresentação e aferição de um modelo iterativo que visa estimar, a determinados níveis de probabilidade, valores mais criteriosos de evapotranspiração de referência que possam ocorrer em uma determinada localidade.

\section{METODOLOGIA}

Numerosos estudos vêm sendo conduzidos ao longo do tempo apresentando indícios de que a ocorrência do parâmetro "evapotranspiração de referência", pode ser estimada pela distribuição beta (YAO,1969; FALLS,1973; BISHNOI,1980). Essa função de probabilidade pode ser expressa da seguinte forma (FALLS,1973):

$$
\begin{aligned}
& B(x)=\frac{1}{(b-a)} \cdot \frac{\Gamma(p+q)}{\Gamma(p) \cdot \Gamma(q)} \cdot\left(\frac{x-a}{b-a}\right)^{p^{-1}} \\
& \left(1-\frac{x-a}{b-a}\right)^{q^{-1}}
\end{aligned}
$$


onde " $\mathrm{a}$ " $\mathrm{e}$ " $\mathrm{b}$ " correspondem a menor e maior valor da série de dados, respectivamente, T corresponde ao símbolo da função gama, "p" c " $q$ " são parâmetros da distribuiça beta, " $x$ " é um valor qualquer da variável em análise. Neste estudo, " $x$ " corresponderia entro ao valor da evapotranspiraçåo potencial (Eto) compreendido no intervalo $[a, b] d a$ série de dados analisadas.

A estimativa dos parámetros " $p$ " $e$ " $q$ " pode ser obtida a partir da utilização do método dos momentos (PEARSON,1934), onde:

$$
\begin{gathered}
p=\frac{\mu_{1} \cdot\left(\mu_{1}-\mu_{2}\right)}{\left[\mu_{2}-\left(\mu_{1}\right)^{2}\right]} \\
q=\frac{\left(1-\mu_{1}\right) \cdot\left(\mu_{1}-\mu_{2}\right)}{\left[\mu_{2}-\left(\mu_{1}\right)^{2}\right]}
\end{gathered}
$$

sendo:

$$
\begin{aligned}
& \mu_{1}=\frac{\sum_{i=1}^{\prime} x_{i}}{N} \\
& \mu_{2}=\frac{\sum_{i=1}^{j} x_{t}^{2}}{N}
\end{aligned}
$$

O termo $\mu_{1}$ corresponde ao momento de ordem 1 para a variável $x, \mu_{2}$ corresponde ao momento de ordem 2 para a variável $x$, c " $N$ " corresponde ao número total de dados que compóe a série analisada

A estimativa da função gama, $\Gamma(\alpha)$, onde $\alpha$ representa uma variável qualquer, ć originária de uma aplicação original da transformada de Laplace, tendo o seguinte formato:

$$
\Gamma(\alpha)=\int_{0}^{x} e^{-x} x^{\alpha-1} d x
$$

para $0 \leq X / \infty$.

Integrando-se a equaçăo por partes, encontra-se a seguinte relação:

$$
\Gamma(\alpha)=(\alpha-1) \cdot \Gamma(\alpha-1)
$$

relação essa que ainda requer transformaçóes para a estimativa da referida funçăo. Nesse caso em particular, existe a possibilidade da utilização de propriedades para a estimativa da função gama (SPIEGEL, 1985), onde:

$$
\Gamma(\alpha)=\frac{\Gamma(\alpha+1)}{\alpha}
$$

ou

$$
\Gamma(\alpha)=(\alpha-1) !
$$

quando $\alpha$ corresponde à um número inteiro positivo, tem-se:

$$
\Gamma(n+1)=n !
$$

Visto a necessidade de determinadas aproximações matemáticas para o cálculo da função , $\Gamma(\alpha)$, ABROMOWITZ \& STEGUN (1970) citam a aproximaçăo polinomial sugerida por Hatings Jr., onde $\Gamma(\alpha+1)$ é estimado por um polinômio de oitavo grau. Para $0 \leq \alpha \leq 1$ tem-se:

$$
\begin{aligned}
& \Gamma(\alpha+1)=1+b_{1} \alpha+b_{2} \alpha^{2}+b_{3} \alpha^{3}+\ldots \\
& \ldots+b_{8} \alpha^{8}+\epsilon(\alpha)
\end{aligned}
$$

sendo:

$|\in(\alpha)| \leq 3,0 \times 10^{-7} ; \quad b_{1}=-0,577191652$
$b_{2}=0,988205891 ; \quad b_{3}=-0,897056937$
$b_{4}=0,918206857 ; b_{3}=-0,756704078$
$b_{6}=0,482199394 ; \quad b_{7}=-0,193527818 ; \quad c$
$b_{8}=0,035886834$

E importante salientar que a distribuição beta, na forma como é apresentada na equação 1 deve ser adimensionalizada para um intervalo compreendido entre $[0,1]$ para que seja possivel a estimativa dos valores de probabilidade. Neste caso, tem-se que:

$$
x^{\prime}=\frac{x-a}{b-a}
$$

sugerindo que a função de densidade da distribuiçăo beta assuma a seguinte forma:

$$
B\left(x^{\prime}\right)=\frac{\Gamma(p+q)}{\Gamma(p) \cdot \Gamma(q)} \cdot x^{, p-1} \cdot\left(1-x^{\prime}\right)_{(10)}^{q-1}
$$

onde $0 \preceq x^{\prime} \preceq 1$, para $p>1$ e $q>1$. 
A integração da equação 10 , que resulta na estimativa da probabilidade, $\mathrm{P}(\mathrm{x})$, de ocorrência do evento não é facilmente solúvel pelos métodos elementares do cálculo diferencial. Para o modelo proposto foi utilizado a integração numérica da função de densidade através da estimativa de áreas entre dois pontos ' $x$ ' consecutivos e muito próximos, aplicando-se a regra dos trapézios.

Baseando-se na teoria apresentada para a estimativa de valores do elemento meteorológico à diversos niveis de probabilidades, procurou-se desenvolver um modelo iterativo cuja estrutura é apresentada na figura 1. Para utilização mais prática, este modelo foi codificado em linguagem Pascal no ambiente Turbo desenvolvido pela Borland International, para microcomputadores da linha IBMPC e compativeis, cuja listagem é apresentada em anexo.

Este modelo foi elaborado e aferido com base nos registros médios de evapotranspiração de referência, estimados para uma série de $\mathbf{3 0}$ anos na região de Piracicaba, São Paulo, situada à latitude de $22^{\circ} 42^{\prime} 30^{\prime \prime} \mathrm{S}$, longitude $47^{\circ} 25^{\prime} 00^{\prime \prime} \mathrm{W}$ e altitude de 580 metros, para o mês de março.

$O$ valor da evapotranspiração a ser considerado no dimensionamento de sistemas de irrigação, depende da duração do período de máxima exigência hidrica da cultura (SAAD,1990). Entretanto, deve-se salientar que esse valor pode ser considerado numa faixa entre 10 a 30 dias. JENSEN (1974), sugere que esse período deve ser considerado de duas a três semanas. SAAD (1990) comenta que o uso de estimativas médias de periodos reduzidos (5 dias) podem conduzir à superestimativas de dimensionamentos, ao passo que em périodos maiores (mensais), há o risco de subdimensionamento do projeto.

Neste contexto, optou-se pela utilização de dados médios de evapotranspiração ajustados em períodos quinzenais. Para cada período, procurou-se estimar os valores de evapotranspiração em diversos níveis de probabilidade, assim divididos: 5,10 , $25,30,40,50,60,70,7580,90$ e $95 \%$, cujos resultados encontrados são apresentados à seguir:

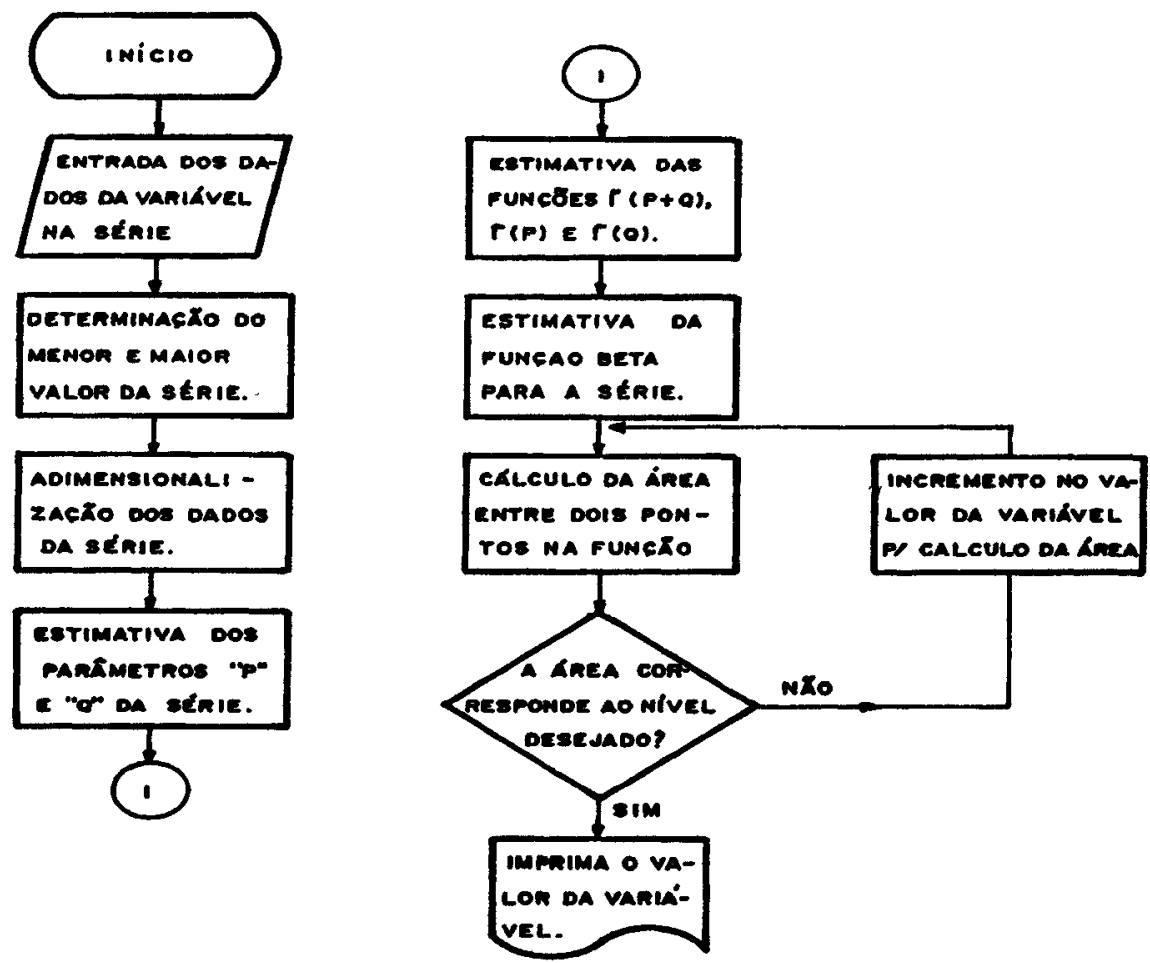

Figura 1 - Diagrama logico de fluxo do modelo iterativo proposto 


\section{RESULTADOS E DISCUSSÕES}

Os valores de evapotranspiração de referência dentro dos niveis de probabilidades sugeridos, para os quinze primeiros dias do mês de março em uma série de 30 anos para a região de Piracicaba, são apresentados na TABELA 1. Como forma de aferição do modelo iterativo proposto, procurou-se comparar os dados estimados, com os obtidos por SAAD (1990) para o período em questão, comparação esta efetivada através do teste de Kolmogorov-Smirnov (CAMPOS, 1983).

Este teste baseia-se na comparação entre uma distribuição conhecida $\mathrm{F}(\mathrm{x})$ que no presente caso corresponde às distribuições encontradas por SAAD (1990), à dados provenientes de uma distribuição estimada $F^{\prime}(x)$, correspondendo aqui às frequências obtidas através dos dados estimados pelo modelo iterativo proposto Os dados estımados por cada estudo são apresentados na TABELA 1.

TABELA 1 - Evapotranspiração de referência em função dos diversos niveis de probabilidade para períodos de quinze dias no mês de março, para Piracicaba-SP. - la. quinzena do mês

\begin{tabular}{ccc}
\hline \hline \multirow{2}{*}{$\begin{array}{c}\text { Niveis } \\
\text { de } \\
\text { Probabilidade }\end{array}$} & \multicolumn{2}{c}{ Evapotranspiração de Referência (mm/dia) } \\
\cline { 2 - 3 } & Modelo Iterativo & SAAD (1990) \\
\hline 5 & 33 & 32 \\
10 & 3.5 & 35 \\
25 & 40 & 40 \\
30 & 41 & 41 \\
40 & 43 & 43 \\
50 & 45 & 45 \\
60 & 47 & 47 \\
70 & 49 & 49 \\
75 & 50 & 51 \\
80 & 51 & 53 \\
90 & 53 & 54 \\
95 & 53 & \\
\hline \hline
\end{tabular}

Como pode ser observado através da TABELA 1 o processo iterativo utilizado gerou resultados praticamente similares aos encontrados por SAAD (1990), para o periodo dos quinze prımeiros dias do mês de março, verificando-se então um allo grau de ajuste nos dados do modelo proposto As diferenças decimass apresentadas entre alguns niveis de probabilidade devem-se, necessariamente, às aproximações utilizadas por metodologias diferentes de estimati$\mathrm{va}$, o que não compromete o resultado final

Utilizando-se o teste de KolmogorovSmırnov para analizar o grau de ajuste das frequências dos dados acima descritos, não verificou-se ao nível de $5 \%$ de probabilidade diferenças estatistıcas entre os dois estudos realizados.
Essa mesma tendêncla pode ser observada através da TABELA 2, onde são apresentados os valores estimados de evapotranspiração de referência para a segunda quinzena do mês de março, à diversos niveis de probabilidade para a região de Piracicaba.

Em se tratando de agricultura irrigada, na estimativa do suprimento de água é comum adotar o conceito de precipitação dependente, isto é, a quantidade de precipitação que se espera ocorrer com uma probabilidade de $75 \%$ (DOORENBOS \& PRUIT, 1976). Tal conceito pode então também ser aplıcado à determinação da chamada "evapotranspiração de dimensionamento", obtida ao nível de $75 \%$ de probabilidade, dentro de uma série de dados. 
TABELA 2 - Evapotranspiração de referência em funçăo dos diversos niveis de probabilidade para períodos de quinze dias do mês de março, para Piracicaba-SP. - 2a. quinzena do mês.

\begin{tabular}{ccc}
\hline \hline $\begin{array}{c}\text { Niveis } \\
\text { de } \\
\text { Probabilidade }\end{array}$ & \multicolumn{2}{c}{ Evapotranspiração de Referência (mm/dia) } \\
\cline { 2 - 3 } 5 & Modelo Iterativo & SAAD (1990) \\
10 & 3.7 & 3.6 \\
25 & 3.8 & 3.8 \\
30 & 4.1 & 4.1 \\
40 & 4.2 & 4.2 \\
50 & 43 & 4.3 \\
60 & 4.4 & 4.5 \\
70 & 4.6 & 4.6 \\
75 & 47 & 4.7 \\
80 & 48 & 4.8 \\
90 & 4.8 & 4.8 \\
95 & 5.0 & 5.0 \\
\hline \hline
\end{tabular}

\section{CONCLUSÕES}

O modelo iterativo proposto para estimativa da evapotranspiração de referência, à diversos níveis de probabilidade, facilita na utilização dos procedimentos matemáticos empregados na análise teórica, em função da série de cálculos necessários e envolvidos no processo. Quando comparado com dados catalogados em literatura, os dados estimados apresentaram um alto grau de ajuste, justificando assim sua utilização prática.

\section{REFERÊNCIAS BIBLIOGRÁFICAS}

ABROMOWITZ, M.; STEGUN, I.A. Handbook of mathematical functions. 9.ed. New York: Dover, 1970. $1046 \mathrm{p}$.

BISHNOI, O.P. The behaviour of moisture adequacy index and its utilization for exploiting the agricultural potential in Punjab and Haryana. Mausam, New Delhi, v.31, n.1, p.157-164, 1980.

CAMPOS, H. Estatística experimental nāo paramétrica. 4.ed. Piracicaba: ESALQ, 1983. 349p.

DOORENBOS, J.; PRUIT, W.O. Las necessidades de água de los cultivos. Roma: FAO, 1976. 173p. (Estudio FAO: riego y drenajes, 24).
FALLS, L.W. The Beta distribution: a statical model for world cloud cover Aiabama: NASA, 1973. 6p. (NASA Technical Memorandum, 64714).

JENSEN, M.E. Consumptive use of water and irrigation water requirements. New York: ASCE, 1974. 215p.

PEARSON, $\mathrm{K}$. Tables of the incomplete Beta function. Cambridge: Unjversity Press, 1934. 494p.

SAAD, J.C.C. Estudo das distribuições de frequência da evapotranspiração de referência e da precipitação pluvial para fins de dimensionamento de sistemas de irrigação. Piracicaba, 1990.124 p Dissertação (Mestrado) - Escola Superior de Agricultura "Luiz de Queiroz", Universidade de São Paulo

SAAD, J.C.C.; SCALOPPI, E J. Análise dos principais métodos climatológicos para estimativa da evapotranspiração. In: CONGRESSO NACIONAL DE IRRIGAÇÃO E DRENAGEM, 8., 1988, Florianópolis, Anais... Florianópolis: ABID, 1988. v.2, p.1037-1052.

SPIEGEL, M.R. Estatística. 2.ed. São Paulo, McGraw-Hill do Brasil, 1985. 453p.

YAO, A.Y.M. The $R$ index for plant water requirement. Agricultural Meteorology, Amsterdam, v.6, p.259-273, 1969.

Recebido para publicação em 01.07 .94

Aceito para publicação em 05.04 .95 